\title{
Chronic Uteroplacental Vasculitis
}

National Cancer Institute

\section{Source}

National Cancer Institute. Chronic Uteroplacental Vasculitis. NCI Thesaurus. Code C118157.

A lymphocytic inflammation of the uteroplacental vessels. 